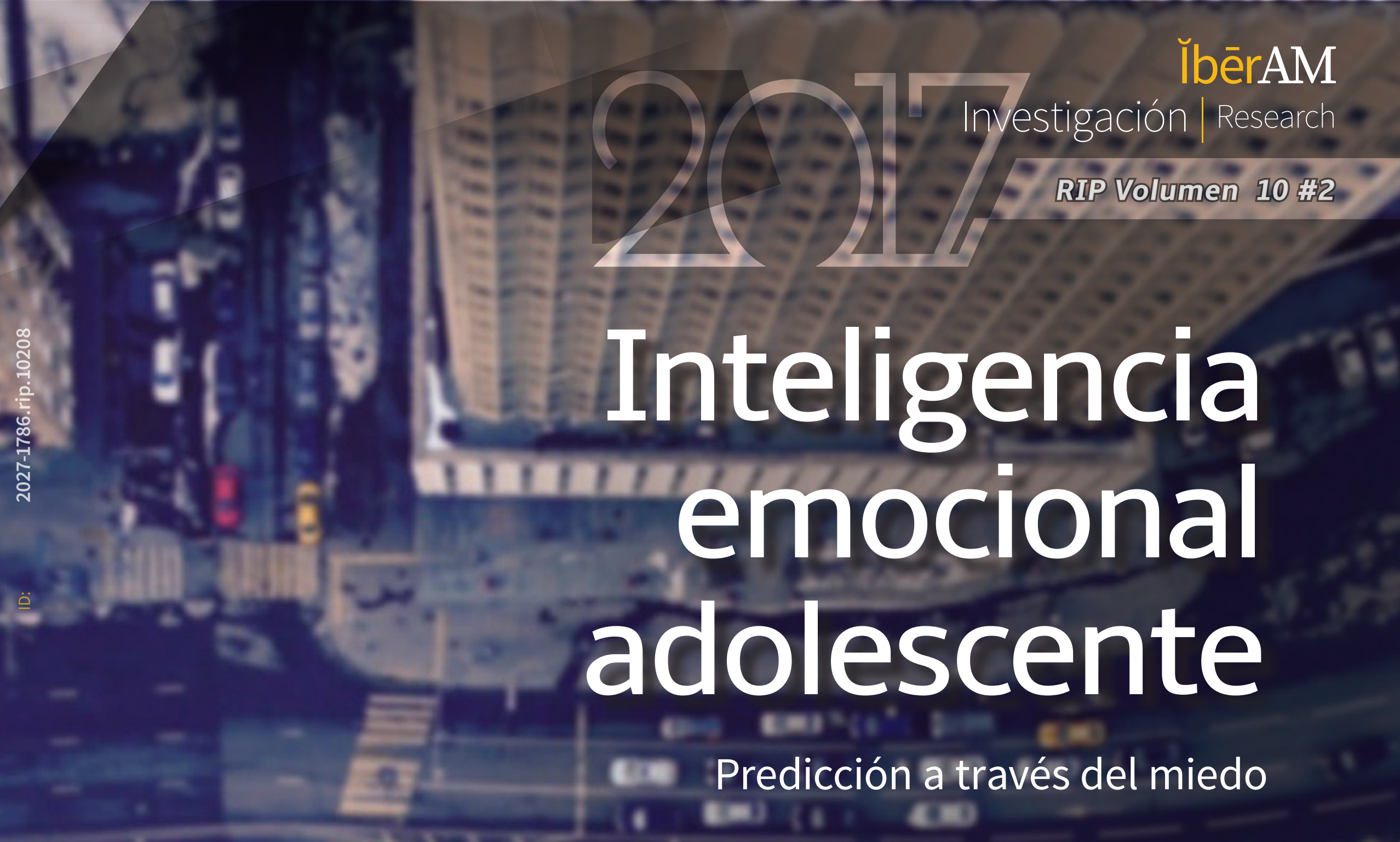

Adolescent emotional intelligence:

Predicting through the fear (c) (1)
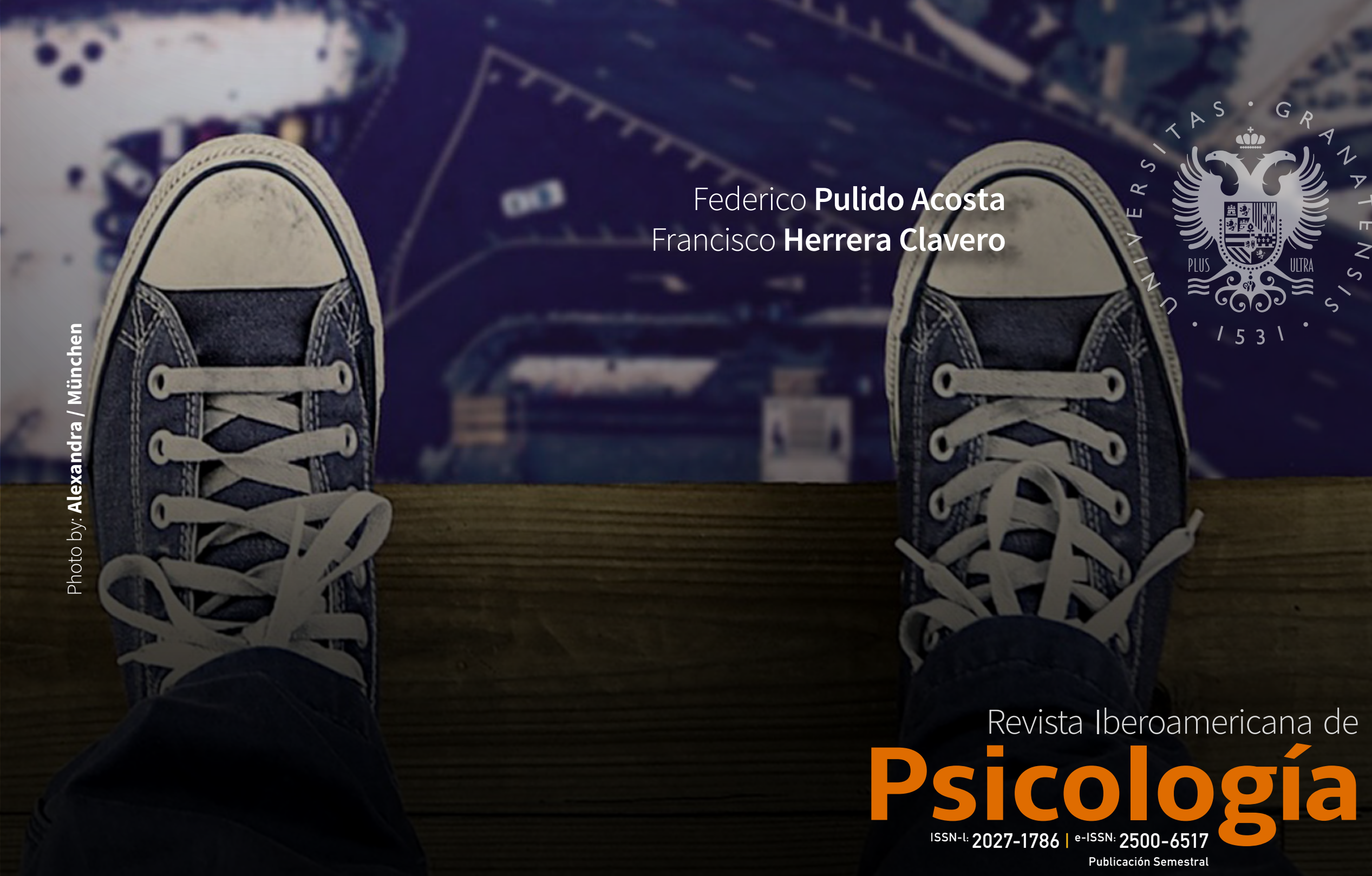


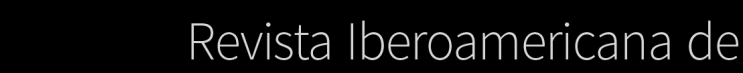 Psicología ISSN-I: 2027-1786 | e-ISSN: 2500-6517 Publicación Semestral}

ID: $\quad$ 2027-1786.rip. 10208

Title:

Subtitle:

Título:

Subtítulo:

Alt Title / Título alternativo:

[en]:

Predicting adolescent emotional intelligence through the fear

[es]: $\quad$ Prediciendo la inteligencia emocional adolescente a través de miedo

Author (s) / Autor (es)

Pulido Acosta, \& Herrera Clavero

Keywords / Palabras Clave:

emoción; miedo; inteligencia emocional; contexto pluricultural

[es]: emotion; fear; emotional intelligence; multicultural context

Submited: 2017-09-23

\section{Resumen}

Este trabajo tiene como objetivo fundamental conocer los predictores de Miedo y la Inteligencia Emocional (IE), así como la influencia entre estos, en alumnos adolescentes de la ciudad de Ceuta. Para ello se contó con la colaboración de 557 participantes de 4 institutos educativos distintos, con edades entre los 12 y 18 años, quienes reflejan las características del contexto pluricultural de la ciudad. De estos 557 estudiantes, el 54.2\% eran niñas y el 45.8\% varones; el 64.5\% de cultura/ religión musulmana y el 35.5\% cristianos.

Como instrumentos de evaluación se emplearon la adaptación de (Ascensio, 2012) del Inventario de Miedo para Niños

(FSSC-II) y un instrumento para medir la Inteligencia Emocional como conjunto de habilidades. Los resultados reflejan niveles medio-bajos de miedo y medio-altos en IE. El predictor del Miedo es el Género, actuando la Edad y la Cultura como predictores de un único factor. Para la IE los predictores son las sociodemográficas de Edad, Cultura/

Religión y Estatus. Se halló una relación estadísticamente significativa entre Miedo y

\section{Abstract}

The main objective of this paper was to know the predictors of Fear and Emotional Intelligence (EI), as well as their combined influence, in teenagers from the city of Ceuta. 557 teenagers, from 4 different highschools, participated, with ages between

12 and 18 years and who clearly reflected the pluricultural context of the city. $45.8 \%$ of the 557 students were boys and 54.2\% were girls; $64.5 \%$ of them were Muslims and 35.5\% were Christians. The Fear Survey Schedule for Children (FSSC-II), adapted by (Ascensio, 2012) for its use in the Spanish language, as

well as an El test, were used to collect the data. The results show mid-low levels on Fear and medium-high in El. The predictor of Fear is the sociodemographic factor 'Gender', and the predictor of El is 'Age', 'Customs/Religion' and 'Socioeconomic' and 'cultural factors'. A statistically significant relationship between Fear and Emotional Intelligence was found.
Citar como:

Pulido Acosta, F., \& Herrera Clavero, F. (2017). Prediciendo la inteligencia emocional adolescente a través del miedo. Revista Iberoamericana de Psicología issn-l:2027-1786, 10 (2), 175-80. Obtenido de: https://revistas.iberoamericana.edu.co/index.php/ripsicologia/article/view/1274
Dr Lic Federico Pulido Acosta

ORCID: $\quad$ 0000-0001-7892-6696

Source I Filiacion:

Universidad de Granada

BIO:

Doctor en Psicología, Universidad de Granada

Licenciado en Psicopedagogía

Maestro

Colaborador en Psicología Evolutiva y de la Educación de la Universidad de Granada, Facultad de Educación y Humanidades de Ceuta.

City I Ciudad:

Ceuta [es]

e-mail:

feanorfede@hotmail.com
DrFrancisco Herrera Clavero

ORCID: $\quad$ 0000-0002-6888-8769

Source I Filiacion:

Universidad de Granada

BIO:

Doctor en Filosofía y Ciencias de la Educación, UNED

Doctor en Psicología, Universidad de Granada

Maestro

Profesor Titular en Psicología Evolutiva y de la Educación de la Universidad de Granada, Facultad de Educación y Humanidades de Ceuta

City I Ciudad

Ceuta [es]

e-mail:

fherrera@ugr.es 


\title{
Inteligencia emocional adolescente Predicción a través del miedo
}

\author{
Adolescent emotional intelligence: Predicting through the fear
}

Federico Pulido Acosta

Francisco Herrera Clavero

\section{Introducción}

El desarrollo de las habilidades y competencias tanto emocionales como sociales es uno de los tantos logros fundamentales que se empiezan a gestar desde la etapa infantil y que perdura hasta la edad adulta. Es por lo tanto imprescindible reflexionar sobre la importancia del desarrollo emocional y la construcción de la personalidad desde el momento del nacimiento, comenzando por su fomento desde la familia y continuando en la escuela (Cruz, 2014), dado que carencias en estos aspecto pueden repercutir de manera incluso determinante, propiciando la aparición de problemas conductuales, conductas disruptivas, escaso bienestar psicológico, rendimiento académico bajo y relaciones interpersonales defectuosas (Gutiérrez \& Expósito, 2015). Consecuentemente, el desarrollo emocional resulta un aspecto indispensable a trabajar si se pretende evitar la aparición de muchas de estas dificultades y si se pretende lograr un desarrollo integral de las personas, para este caso específico, los alumnos adolescentes. En este sentido, muchos de los principales modelos de intervención orientadora centran sus esfuerzos en el desarrollo de programas de educación emocional con independencia de la etapa educativa a la que éstos vayan dirigidos (Pulido \& Herrera, 2015)

En el contexto de la reflexión sobre la importancia de las emociones en el desarrollo personal, social, profesional y académico de las personas, aparece el concepto de Inteligencia Emocional (IE) entendido como la habilidad (o conjunto de habilidades) para manejar o gestionar el propio repertorio emocional, de una manera adecuada y en cierto sentido exitosa. Entre las habilidades específicas que integran la IE (Pulido \& Herrera, 2015), están las habilidades para el auto-conocimiento o conocimiento de sí mismo (entendido como la adecuada identificación de las propias emociones), el autocontrol (manejo o gestión de las propias emociones), la motivación (relacionada con el impulso direccionador para el desarrollo de una tarea), la empatía (capacidad de identificar las emociones en los demás) y habilidades sociales (habilidades que permiten la adecuada interacción con los demás).
En lo que respecta al abordaje de las emociones específicamente, se toman como partida teorías centradas en tres componentes cruciales, a saber: el componente experiencial-cognitivo, el componente fisiológico y el de reacción conductual, de carácter adaptativo (Sroufe, 2000). Desde esta perspectiva, una emoción consiste en el análisis perceptivo-cognitivo de una situación que desencadena un correlato fisiológico y que tiene una función adaptativa al influir en la conducta del sujeto ante el entorno y ante los otros (Pulido, 2016). De acuerdo con esto, una emoción no se puede considerar como positiva o negativa en sí misma, esto lo determinan las sensaciones favorables o desfavorables experimentadas que la acompañan o suceden.

Si bien todas las emociones son relevantes, es importante considerar las consecuencias desfavorables asociadas a estados emocionales de carácter negativo y que pueden dificultar el adecuado desarrollo. Estos estados emocionales negativos pueden afectar el autoconcepto, incidiendo en una falta de aceptación por parte de los demás (Delgado, Inglés, \& García Fernández, 2013), también pueden ser responsables de problemas académicos (Ranøyen, Jozefiak, Wallander, Lydersen, \& Indredavik, 2014), causar problemas de aprensión, tensión o intranquilidad, depresión, desesperanza, tristeza, ambivalencia e hiperactividad e incluso pensamientos suicidas (Bazán López, Olórtegui Malaver, \& Huayanay Falconí, 2016).

Con miras a alcanzar el objetivo trazado, el presente trabajo se centrará en la emoción del miedo, identificándola como una emoción de carácter negativo a raíz de las sensaciones desfavorables que la acompañan o que desencadena, y distinguiéndola de otros estados emocionales como la ansiedad o las fobias, en consonancia con (Gómez Ortiz, Casas, \& Ortega Ruiz, 2016) 


\section{Marco Teórico}

En la constelación de las emociones hay diversas variables que ejercen influencia, como la edad, la cultura y el género, por mencionar algunas. La influencia de la edad sobre las manifestaciones emocionales resulta muy clara y evidente en una disminución progresiva del número de cosas y eventos que causan miedo, así como en una disminución de la intensidad de los mismos, realidad que se mantiene hasta el momento en que se alcanza la etapa adulta (Knappe, Sasagawa, \& Creswell, 2015; Pulido, 2016). La cultura de referencia, cultura de origen, tiene una gran influencia en las relaciones afectivas y en la constitución de diferentes manifestaciones emocionales, así como en la educación de la competencia emocional (Pulido, 2016). El género también constituye otra variable determinante en la incidencia de los miedos (Pulido, 2016), y a este respecto tienden a destacar las mujeres.

Considerando todas las habilidades, capacidades y variables que influyen las emociones, resulta imprescindible contar con instrumentos adecuados para evaluar la $\boldsymbol{I E}$, que tengan en cuenta la interacción entre las emociones y el pensamiento, así como las relaciones entre las diferentes dimensiones de la Inteligencia Emocional y la calidad de las relaciones interpersonales (Ruivo \& Paes, 2015), además de ser instrumentos validados transculturalmente, con poblaciones de distintos contextos lingüísticos y culturales. Este tipo de instrumentos, por sus características, se alejan de aquellos propios de los modelos percibidos (autopercepción de la $\boldsymbol{I E}$ ), como los inventarios de autoinforme. Lo anterior, gracias a la propuesta de nuevos modelos de $\boldsymbol{I} \boldsymbol{E}$, desde los cuales se han llevado a cabo diferentes trabajos orientados a evaluar los componentes y habilidades de la misma, y a estudiar cómo dichos componentes funcionan en las personas (Sainz Gómez, Bermejo García, Ferrándiz García, Prieto Sánchez, \& Ruiz Melero, 2015).

Específicamente, la relación que atañe aquí es aquella entre el miedo en tanto emoción de carácter negativo y aquellas capacidades que se han incluido dentro de la Inteligencia Emocional (Ford, Mauss, Troy, Smolen, \&Hankin, 2014; Ordóñez Camblor, y otros, 2014). Las emociones negativas, como el miedo, influyen en el desempeño emocional del individuo y en su competencia social, en tanto las personas que evidencian mayores niveles de miedo están menos abiertas a la interacción con otrosy estas interacciones son imprescindibles para el fortalecimiento de todas las habilidades socio-emocionales. Con la intención de mitigar este tipo de estados emocionales, prevenir sus consecuencias desfavorables, disminuir los problemas que puede devenir de la inadecuada gestión de las emociones y fortalecer el desarrollo socioemocional de las personas, se aboga por el desarrollo de las habilidades emocionales integradas en la Inteligencia Emocional, entre las que se encuentra la adecuada regulación del repertorio emocional.

Es así como queda justificada la relevancia social del presente trabajo, el cual busca conocer los predictores del miedo y de la IE en una muestra adolescentes escolarizados en institutos de la ciudad de Ceuta, la cual se distingue por su diversidad cultural y social (Balongo \& Mérida, 2016) Indagar por las relaciones entre el miedo y la IE permitiría comprobar si los miedos pueden actuar como predictores de la IE, al igual que el nivel de IE como predictora del miedo, de tal manera que puedan emplearse para potenciar el desarrollo de habilidades emocionales, con la intención de potenciar el desempeño emocional del sujeto y favorecer las competencias emocionalmente ajustadas que favorecerían la disminución progresiva de los estados emocionales de carácter negativo (Gutiérrez \& Expósito, 2015; Caballero Dominguez, Suarez Colorado, \& Bruges Carbono, 2015).

Esto también conlleva a comprender mejor las variables intervinientes en ambas, a saber: el género, la edad, la cultura y el estatus socio-económico y cultural. A partir de la revisión bibliográfica realizada se esperan resultados superiores en los niveles de Miedo en función del Género, la Cultura y el Estatus. En IE se esperan obtener diferencias en función de la Edad, fruto del proceso de desarrollo emocional, aumentando los niveles a medida que asciende la edad del sujeto. También se esperan obtener niveles diferentes de IE entre los sujetos con niveles más elevados de Miedo. Todos ellos funcionarán como predictores de sus respectivas variables.

\section{Método}

\section{Participantes}

Para llevar a cabo esta investigación se seleccionó una muestra de 557 estudiantes adolescentes (Tabla 1) quienes reflejan las características del contexto pluricultural de Ceuta. Estos estudiantes pertenecían a cuatro institutos educativos de esta ciudad, cuyos nombres fueron reemplazados por nombres en clave con el fin de preservar la identidad de los mismos. Así pues, el $\mathbf{2 9 . 3 \%}$ de la muestra provenía del Instituto Naranja, el $\mathbf{2 9 . 4 \%}$ del Blanco, el $\mathbf{2 1 . 2 \%}$ del Amarillo y el restante $\mathbf{2 0 . 1 \%}$ del Negro.

Los participantes se seleccionaron por el método de muestreo incidental o casual. Se seleccionó de manera aleatoria un curso (desde primer grado de ESO hasta segundo de Bachillerato) de cada uno de los institutos, y fueron aplicados ambos cuestionarios durante la primera hora de clase. El margen de error en la muestra fue del $\mathbf{3 \%}$.

La muestra se distribuyó de la siguiente manera: el $\mathbf{1 7 . 1 \%}$ de los alumnos era de primero, el $\mathbf{1 5 . 4 \%}$ de segundo, el $\mathbf{2 0 . 3} \%$ de tercero y el $\mathbf{2 1 . 5 \%}$ de cuarto de ESO. Respecto a los Bachilleratos, el $\mathbf{1 2 . 9 \%}$ de primero y el $\mathbf{1 2 . 7 \%}$ de segundo. En función de la variable género el $\mathbf{4 5 . 8 \%}$ eran chicos y el resto, $\mathbf{5 4 . 2} \%$, chicas. En términos de la cultura (que se para este caso específico corresponde con la religión) el grupo mayoritario $(\mathbf{6 4 . 5} \%$ de la muestra) fue de participantes pertenecientes a la cultura/religión musulmana, mientras que el $\mathbf{3 5 . 5 \%}$ de la muestra pertenecía a la cultura/religión cristiana. Finalmente, atendiendo al estatus el $\mathbf{6 . 3 \%}$ de la muestra se identificó como de nivel bajo, el $\mathbf{4 . 8 \%}$ se identificó como perteneciente a un nivel alto, el $\mathbf{3 8 . 1 \%}$ al estatus medio-bajo y el $\mathbf{5 0 . 8} \%$ al medio.

Tabla 1 Muestra en función de variables sociodemográficas

\begin{tabular}{|c|c|c|c|c|c|}
\hline \multirow[t]{2}{*}{ Variables } & & \multicolumn{4}{|c|}{ Media descriptiva } \\
\hline & & Media & D.T. & Rango & \\
\hline \multicolumn{2}{|c|}{ Edad } & $\mathrm{N}=557$ & 14.93 & 1.696 & $12-18$ \\
\hline & & & & N & $\%$ \\
\hline \multirow{2}{*}{ Sexo } & Chicos & & & 255 & 45.8 \\
\hline & Chicas & & & 302 & 54.2 \\
\hline \multirow{2}{*}{ Cultura/Religión } & Cristianos & & & 198 & 35.5 \\
\hline & Musulmanes & & & 359 & 64.5 \\
\hline \multirow{5}{*}{ Estatus } & Bajo & & & 35 & 6.3 \\
\hline & Medio-Bajo & & & 212 & 38.1 \\
\hline & Medio & & & 283 & 50.8 \\
\hline & Alto & & & 27 & 4.8 \\
\hline & & & \multicolumn{3}{|c|}{ Fuente: elaboración propi } \\
\hline
\end{tabular}

\section{Instrumentos}

Como instrumento para evaluar el miedo se empleó el Inventario de Miedos para Niños (FSSC-II), en su adaptación al español (Ascensio, y otros, 2012). Su alta fiabilidad queda reflejada por un Alpha de Cronbach 
de $\mathbf{0 . 9 5 7}$. Para la clasificación de los ítems por factores, se obtuvo los 5 especificados en el cuestionario original, agrupados de la siguiente forma: Miedo a los Animales y al Peligro (reflejó una varianza explicada del 12.37\%), Miedo a la Muerte (integró un total de 26 ítems, los cuales ostentaban una varianza explicada del 9.99\%), Miedo a lo Desconocido (7.34\%), Miedos Escolares (6.52\%) y Miedos Médicos (3.85\%). La suma de la varianza explicada de los cinco factores fue de $\mathbf{4 0 . 0 7 \%}$. Cada uno de los participantes contestó al cuestionario durante la primera hora de clase, en una única sesión, después de recibir información sobre el modo de contestar cada uno de los ítems.

La evaluación de la IE se llevó a cabo a través de un test elaborado. Para realizar el proceso de adaptación se siguió una estructura que contenía apartados para la identificación de emociones en gestos faciales, en diferentes fotografías, la utilidad de diferentes emociones en situaciones determinadas, la selección de la emoción que más se ajustaba a cada caso, la respuesta a cuestiones emocionales acordes a determinadas historias y la relación de diferentes emociones con acciones concretas. Las diferentes secciones o apartados se ajustaron en términos de dificultad de acuerdo al alumnado que contestaría. La fiabilidad de este instrumento quedó reflejada por un Alpha de Cronbach de $\mathbf{0 . 8 1 3}$ y sus ítems se clasificaron en cinco factores: autoconcepto (con el $\mathbf{5 . 8 \%}$ de la varianza explicada total), empatía

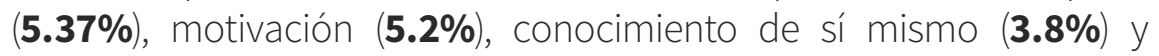
autocontrol (3.7\%). Todo ello supone un total de 66 ítems, que dan una varianza explicada total de $\mathbf{2 3 . 8 7 \%}$.

A los estudiantes se les informó sobre el objetivo del estudio, la manera de proceder y el respeto de su confidencialidad. Los cuestionarios fueron distribuidos una vez se contó con la participación voluntaria de los estudiantes con las autorizaciones pertinentes por parte de la Dirección Provincial de Educación y de cada uno de los diferentes centros educativos. Todos los cuestionarios fueron entregados en los diferentes centros educativos por el investigador, en presencia del profesor de cada aula.

Una vez recabados los datos se elaboró una base de datos y se procedió con el análisis descriptivo, haciendo uso de tablas de contingencia para plasmar los porcentajes alcanzados por la muestra general y en función de las variables sociodemográficas.

Con el fin de comprobar los predictores se muestran los resultados de la regresión múltiple paso a paso o stepwise, considerando como variable criterio la IE, el miedo, así como cada uno de los factores que forman parte de ambos. Como predictoras se utilizaron las sociodemográficas y la que no actuaba como variable criterio.

Elanálisiscompleto permitiólacomprobacióndel comportamiento de la muestra desde la perspectiva de las variables sociodemográficas, así como la interacción entre las variables de estudio.

\section{Resultados}

La respuesta más frecuente fue la opción poco miedo, elegida por poco más de la mitad de la muestra (50.4\% de los participantes), seguida por la opción bastante miedo elegida por el $\mathbf{3 1 . 2 \%}$ de los participantes. Por su parte las opciones menos escogidas por los respondientes fueron la opción más baja (16.2\%) y de mucho miedo (2.2\%).

Los resultados consignados en la Tabla 2 muestran que el Miedo Total está bajo la influencia de la variable Género, descartando el resto de variables. El porcentaje de varianza total es de $\mathbf{2 1 . 2 \%}\left(R^{2}=.212\right)$. El poder determinante de la única variable influyente, el Género, explica el $\mathbf{4 6} \%$ de la varianza total $(\beta=.460)$. Las mujeres (chicas) obtuvieron puntuaciones más altas, en tanto se asoció el valor 1 con los varones y el 2 con las mujeres.

Tabla 2 Regresión Miedo Total

\begin{tabular}{ccccc} 
Variables & $\mathrm{B}$ & $\mathrm{Q}$ & $\mathrm{t}$ & $\mathrm{p}$ \\
\hline Constante General & 42.649 & & 8.689 & .000 \\
Género & 36.986 & .460 & 12.206 & .0000
\end{tabular}

Miedo Total= 42.649 + 36.986 (Género); $R=.460 ; R^{2}=.212 ; F=148.993 ; p=.0000$; Fuente: elaboración propia

Los resultados dela regresión múltiple(Tabla3) indican queelfactor Miedo a los Animales y al Peligro está bajo la influencia de la variable Género y Cultura/Religión, que en conjunto dan cuenta del $\mathbf{2 8 . 5 \%}$ de la varianza en este factor $\left(R^{2}=.285\right)$. El mayor poder determinante lo tiene el Género, que explica el $\mathbf{5 0 \%}$ de la varianza ( $\beta=.500)$. Los valores positivos en Género indican mayores puntuaciones entre las mujeres (chicas), mientras que la Cultura/Religión indica que son los musulmanes quienes evidencian niveles superiores en esta emoción (los valores positivos indican esto, ya que la cultura cristiana se asoció al valor numérico 1 y la musulmana a la 2).

Tabla 3 Regresión Miedo Animales

\begin{tabular}{ccccc}
\hline Variables & $\mathrm{B}$ & $\mathrm{Q}$ & $\mathrm{t}$ & $\mathrm{P}$ \\
\hline Constante General & -7.607 & & -3.529 & .000 \\
Género & 12.989 & .500 & 13.878 & .0000 \\
Cultura/Religión & 4.452 & .165 & 4.571 & .000
\end{tabular}

Miedo Animales= -7.607 + 12.989 (Género) + 4.452 (Cultura/Religión); R=.533; $\mathrm{R} 2=.285 ; \mathrm{F}=109.944 ; \mathrm{p}=.0000 ;$ Fuente: elaboración propia

De acuerdo a los datos arrojados por la regresión (Tabla 4) es posible ver que el Miedo a la Muerte está bajo la influencia de las variables Género, Inteligencia Emocional y Curso/Edad que, en conjunto, dan cuenta del $\mathbf{1 7 . 7 \%}$ de la varianza en este factor de Miedo $\left(R^{2}=.177\right)$. La variable más determinante resultó ser el Género, que por sí solo explica el $\mathbf{3 7 . 1 \%}$ de la varianza ( $\beta=.371)$. El valor de la variable Género en la función volvió a ser positivo, lo que indica que las mujeres (chicas) tienen puntuaciones más altas. La IE es directamente proporcional al Miedo a la Muerte, mientras que la edad (Curso/Edad) es inversamente proporcional a esta.

Tabla 4 Regresión Miedo Muerte

$\begin{array}{ccccc}\text { Variables } & \mathrm{B} & \mathrm{X} & \mathrm{t} & \mathrm{P} \\ \text { Constante General } & 24.511 & & 5.539 & .000 \\ \text { Género } & 11.783 & .371 & 9.562 & .000 \\ \text { Inteligencia Emocional } & .136 & .186 & 4.340 & .000 \\ \text { Curso/Edad } & -.984 & -.100 & -2.337 & .020\end{array}$

Miedo Muerte $=24.511+11.783$ (Género) $+.136($ IE) -.984 (Curso/Edad); $\mathrm{R}=.421 ; \mathrm{R}^{2}=.177 ; \mathrm{F}=39.599 ; \mathrm{p}=.0000 ;$ Fuente: elaboración propia

En la Tabla 5 se consignan los resultados que permiten evidenciar que el Miedo a lo Desconocido está bajo la influencia de la variable Género, que da cuenta del $\mathbf{1 6 . 2} \%$ de la varianza $\left(R^{2}=.162\right)$. Como único predictor está el Género que ejerce el mayor poder determinante, explicando el $\mathbf{4 0 . 3 \%}$ de la varianza total $(\beta=.403)$. Los hombres (chicos) volvieron a presentar puntuaciones inferiores en este factor.

Tabla 5 Regresión Miedo Desconocido

$\begin{array}{ccccc}\text { Variables } & \mathrm{B} & \mathrm{Q} & \mathrm{t} & \mathrm{P} \\ \text { Constante General } & 1.488 & & 1.340 & .181 \\ \text { Género } & 7.097 & .403 & 10.356 & .0000\end{array}$

Miedo Desconocido= 1.488 + 7.097 (Género); R=.403; R²=.162; F=107.240; $\mathrm{p}=.0000$; Fuente: elaboración propia

Los resultados de la regresión (Tabla 6) permiten predecir la puntuación del factor Miedos Escolares conociendo el Género del sujeto. Esto da el $\mathbf{5 \%}$ de la varianza total en este factor $\left(R^{2}=.050\right)$. El Género explica el $\mathbf{6 2 . 7 \%}$ de la varianza total ( $\beta=.627$ ). De nuevo, son las 


\section{inteligencia emocional adolescente}

Predicción a través del miedo

mujeres (chicas) las que obtendrá resultados superiores de seguirse la función que aparece a continuación.

Tabla 6 Regresión Miedos Escolares

\begin{tabular}{ccccc}
\hline Variables & $\mathrm{B}$ & $\mathrm{Q}$ & $\mathrm{t}$ & $\mathrm{P}$ \\
\hline Constante General & 9.729 & & 9.583 & .000 \\
Género & 3.396 & .627 & 5.418 & .000
\end{tabular}

Miedos Escolares= 9.729 + 3.396 (Género); $R=.224 ; R^{2}=.050 ; F=29.358 ; p=.0000$; Fuente: elaboración propia

Los cálculos de regresión reflejan que se pueden anticipar los Miedos Médicos si se conocen las variables Género e Inteligencia Emocional. Estas dos variables predictoras representan el $\mathbf{4 \%}$ de la varianza en Miedos Médicos ( $\left.\mathrm{R}^{2}=.040\right)$. El mayor poder determinante vuelve a tenerlo el Género, que por sí solo explica el $\mathbf{1 8 . 4 \%}$ de la varianza total ( $\beta=$.184). Las mujeres (chicas) vuelven a tener niveles superiores. Los Miedos Médicos y la IE son inversamente proporcionales en tanto los niveles decrecen en este factor a medida que ascienden las puntuaciones en IE (Tabla 7).

Tabla 7 Regresión Miedos Médicos

\begin{tabular}{|ccccc|}
\hline Variables & $\mathrm{B}$ & $\mathbb{Q}$ & $\mathrm{t}$ & $\mathrm{P}$ \\
\hline Constante General & 2.964 & & 3.531 & .000 \\
Género & 1.217 & .184 & 4.388 & .000 \\
Inteligencia Emocional & -.015 & -.096 & -2.291 & .022 \\
\hline
\end{tabular}

Miedos Médicos= 2.964 + 1.217 (Género) - .015 (IE); R=.199; R²=.040; F=11.397; $\mathrm{p}=.0000$; Fuente: elaboración propia

El principal predictor del Miedo es el Género. Las mujeres (chicas) evidencian un nivel más alto de miedo que los hombres (chicos), observación que se apoya en las medias de ambos (79.6 los hombres y $\mathbf{1 1 6 . 7}$ las mujeres). Las diferencias son significativas en el en el Miedo Total $(\mathrm{p}=.000)$, Miedo a los Animales $(\mathrm{p}=.000)$, Miedo a la Muerte $(\mathrm{p}=.000)$, Miedo a lo Desconocido $(\mathrm{p}=.000)$, los Miedos Escolares $(\mathrm{p}=.000)$ y Miedos Médicos $(\mathrm{p}=.000)$. En todos ellos, las mujeres (chicas) reflejan niveles más altos.

El curso/grado escolar aparece como predictor en un factor. El grupo en el que se registran los mayores niveles de Miedo es precisamente en el de los alumnos con mayor edad $\left(2^{\circ}\right.$ de Bachillerato con una media de 106.04), algo que resulta inesperado. Este viene seguido por primer grado $1^{\circ}$ de ESO (105.6), seguido de $1^{\circ}$ de Bachillerato (100.2). Las diferencias no son muy evidentes y todos los grupos se registran en niveles medio-bajos, dado que las diferencias no son significativas para el Miedo Total $(\mathrm{p}=.160)$, el Miedo a los Animales $(\mathrm{p}=.228)$, Miedo a la Muerte ( $\mathrm{p}=.071)$, Miedo a lo Desconocido $(\mathrm{p}=.383)$, los Miedos Escolares ( $p=.357$ ) y los Miedos Médicos ( $p=.369)$.

La situación anterior se repite con la Cultura. El alumnado perteneciente a la Cultura/Religión musulmana manifestó niveles más elevados de miedo (la media de los cristianos fue $\mathbf{9 4 . 5 5}$ y los musulmanes alcanzaron $\mathbf{1 0 2 . 6 5}$ ). Los resultados demuestran que la cultura influye en el Miedo Total ( $p=.022)$, en el Miedo a los Animales y en Miedo al Peligro ( $\mathrm{p}=.000)$. Sin embargo, no fue significativa para el Miedo a la Muerte $(p=.781)$, Miedo a lo Desconocido $(p=.099)$, los Miedos Escolares ( $p=.052)$ y los Miedos Médicos $(p=.231)$. Los niveles más altos de miedo se registraron en los musulmanes.

Resulta destacable que ninguno de los encuestados (0\%) reflejó niveles muy bajos en la variable IE. La opción mayoritaria evidencia un alto nivel de $\boldsymbol{I} \boldsymbol{E}$, correspondiente al $\mathbf{7 2 . 3 \%}$ de la muestra. El resto de encuestados se distribuyeron de manera muy similar entre la opción de mayor nivel de IE (14.2\%) y poca IE $(\mathbf{1 3 . 5 \% ) . ~}$

Los datos consignados en la Tabla 8 permiten calcular la Inteligencia Emocional total del sujeto en función de las variables Curso/Edad, Estatus, Miedo Total y Cultura/Religión. El Curso/Edad es directamente proporcional a las puntuaciones en IE. Son positivos

también los valores del Estatus y el Miedo Total. A estatus más elevado y mayores niveles de Miedo, son más altas las puntuaciones en IE. El único valor negativo corresponde a la Cultura/Religión, lo que indica que son los cristianos los que obtuvieron resultados superiores. Todas las variables predictoras dan cuenta del $\mathbf{3 4 . 6 \%}$ de la varianza en la IE $\left(R^{2}=.346\right)$. El mayor poder determinante lo ejerce el Curso/Edad, que por sí solo explica el $\mathbf{4 7 . 1 \%}$ de la varianza en IE $(\beta=.471)$.

Tabla 8 Regresión IE

\begin{tabular}{|ccccc|}
\hline Variables & $\mathrm{B}$ & $\mathrm{Q}$ & $\mathrm{t}$ & $\mathrm{P}$ \\
\hline Constante General & 30.576 & & 4.483 & .000 \\
Curso/Edad & 6.355 & .471 & 13.547 & .0000 \\
Estatus & 11.460 & .363 & 10.042 & .0000 \\
Miedo Total & .049 & .090 & 2.590 & .010 \\
Cultura/Religión & -3.894 & -.086 & -2.387 & .017
\end{tabular}

Inteligencia Emocional $=30.576+6.355($ Curso/Edad $)+11.460($ Estatus $)+.049$ (Miedo Total) - 3.894 (Cultura/Religión); $R=.588 ; R^{2}=.346 ; F=72.876 ; p=.0000$; Fuente: elaboración propia

Los resultados resumidos en la Tabla 9 muestran que el Autoconcepto (factor de la IE) está bajo la influencia de las variables Curso/Edad, Estatus, Miedo Total y Cultura/Religión, que en conjunto dan cuenta del $\mathbf{2 2 . 8 \%}$ de la varianza en el Autoconcepto $\left(\mathrm{R}^{2}=.228\right)$. El mayor poder determinante lo ejerce la variable Curso/Edad, que explica el $\mathbf{3 5 . 2 \%}$ de la varianza ( $\beta=$.352). A mayor edad, Estatus y nivel de Miedo, más elevadas son las puntuaciones en este factor de la IE. La Cultura/Religión tiene valores negativos en la función, indicando de nuevo menores puntuaciones entre los musulmanes.

Tabla 9 Regresión Autoconcepto

\begin{tabular}{|c|c|c|c|c|}
\hline Variables & B & 凶 & $\mathrm{t}$ & p \\
\hline Constante General & 7.841 & & 4.607 & .000 \\
\hline Curso/Edad & 1.090 & .352 & 9.312 & .000 \\
\hline Estatus & 1.797 & .248 & 6.307 & .000 \\
\hline Miedo Total & .027 & .217 & 5.763 & .000 \\
\hline Cultura/Religión & -.841 & -.081 & -2.064 & .039 \\
\hline \multicolumn{5}{|c|}{$\begin{array}{r}\text { Autoconcepto }=7.841+1.090(\text { Curso/Edad })+1.797(\text { Estatus })+.027 \text { (Miedo } \\
\text { Total) }-.841 \text { (Cultura/Religión); } R=.478 ; R^{2}=.228 ; F=40.694 ; p=.0000 ; \text { Fuente } \\
\text { elaboración propia }\end{array}$} \\
\hline
\end{tabular}

La Empatía se puede predecir si se conocen los valores de Curso/ Edad, Estatus y Miedo Total, siendo la primera ( $\beta=$.333) la que mayor poder determinante ejerce, con un $\mathbf{3 3 . 3} \%$ de la varianza total, que es del $\mathbf{1 8 \%}\left(R^{2}=\right.$. 180). Todas las variables son directamente proporcionales a la Empatía, aumentando los niveles a medida que aumenta la Edad, el Estatus y las manifestaciones Totales de Miedo. Los resultados quedan consignados en la Tabla 10.

Tabla 10 Regresión Empatía

\begin{tabular}{|ccccc|}
\hline Variables & B & Q & t & p \\
Constante General & 10.724 & & 4.221 & .000 \\
Curso/Edad & 1.757 & .333 & 8.580 & .000 \\
Estatus & 3.143 & .255 & 6.563 & .000 \\
Miedo Total & .032 & .152 & 3.947 & .000
\end{tabular}

Empatía $=10.724+1.757($ Curso/Edad $)+3.143$ (Estatus) +.032 (Miedo Total); $\mathrm{R}=.424 ; \mathrm{R}^{2}=.180 ; \mathrm{F}=40.282 ; \mathrm{p}=.0000$; Fuente: elaboración propia

Tabla 11 Regresión Motivación

$\begin{array}{ccccc}\text { Variables } & \mathrm{B} & \mathrm{Q} & \mathrm{t} & \mathrm{p} \\ \text { Constante General } & -.724 & & -.331 & .741 \\ \text { Curso/Edad } & 1.549 & .337 & 8.781 & .000 \\ \text { Estatus } & 3.211 & .300 & 7.807 & .000 \\ \text { Miedo Total } & -.028 & -.150 & -3.947 & .000\end{array}$

Motivación = $-0.741+1.549($ Curso/Edad $)+3.211$ (Estatus) -0.028 (Miedo Total); $\mathrm{R}=.447 ; \mathrm{R}^{2}=.200 ; \mathrm{F}=45.897 ; \mathrm{p}=.0000$; Fuente: elaboración propia 
La Motivación (Tabla 11) está bajo la influencia de las variables Curso/Edad, Estatus y Miedo Total que, en conjunto, dan cuenta del $\mathbf{2 0} \%$ de la varianza en la Motivación $\left(\mathrm{R}^{2}=.200\right)$. El mayor poder determinante lo vuelve a tener la Edad, que representa el $\mathbf{3 3 . 7 \%}$ de la varianza $(\beta=.337)$. Las variables Curso y Estatus adoptan valores positivos, actuando de la misma manera que en los casos anteriores. El Miedo Total es inversamente proporcional a la Motivación.

Los datos arrojados por la regresión múltiple (Tabla 12) indican que el factor Conocimiento de sí mismo está también relacionado con la variable Curso/Edad, Estatus y Cultura/Religión, que en conjunto dan cuenta del $\mathbf{1 4 . 7 \%}$ de la varianza en este factor $\left(R^{2}=.147\right)$. El mayor poder determinante vuelve a tenerlo la Edad, que explica el $\mathbf{2 8 . 4 \%}$ de esta varianza ( $\beta=.284)$. El Conocimiento de sí mismo es directamente proporcional a la Edad y el Estatus, y es inversamente proporcional a la Cultura/Religión. Esto último indica que son los musulmanes quienes tienen puntuaciones menores en este factor.

Tabla 12 Regresión Conocimiento de sí mismos

$\begin{array}{ccccc}\text { Variables } & \mathrm{B} & \mathrm{Q} & \mathrm{t} & \mathrm{p} \\ \text { Constante General } & 11.201 & & 6.432 & .000 \\ \text { Curso/Edad } & .876 & .284 & 7.151 & .000 \\ \text { Estatus } & 1.586 & .220 & 5.329 & .000 \\ \text { Cultura/Religión } & -1.330 & -.128 & -3.139 & .002\end{array}$

Conocimiento de sí mismos= $11.201+.876$ (Curso/Edad) +1.586 (Estatus) - 1.330 (Cultura/Religión); $R=.384 ; R^{2}=.147 ; F=31.750 ; p=.0000$; Fuente: elaboración propia

Para el Autocontrol, los resultados de la regresión múltiple (Tabla 13) indican que existe influencia del Curso/Edad y el Estatus. Ambas explican el $\mathbf{1 6 . 7 \%}$ de la varianza en este factor de la IE $\left(R^{2}=.167\right)$. El mayor poder determinante lo vuelve a tener la Edad que justifica el $\mathbf{3 4 . 1 \%}$ de la varianza en Autocontrol ( $\beta=$.341). En este caso, ambas variables tienen valores positivos, aumentando las puntuaciones con la Edad y en los Estatus superiores.

Tabla 13 Regresión Autocontrol

\begin{tabular}{ccccc}
\hline Variables & B & Q & t & P \\
\hline Constante General & -.785 & & -.522 & .602 \\
Curso/Edad & 1.113 & .341 & 8.728 & .000 \\
Estatus & 2.069 & .271 & 6.937 & .000
\end{tabular}

Autocontrol= $-.785+1.113$ (Curso/Edad) + 2.069 (Estatus); R=.409; R²=.167; $\mathrm{F}=55.555 ; \mathrm{p}=.0000$; Fuente: elaboración propia

Los resultados consignados en la Tabla 14 muestran que las Habilidades Sociales están bajo la influencia de la variable Miedo Total solamente. Esta da cuenta del $\mathbf{8 . 2} \%$ de la varianza en $\boldsymbol{H H S S}$ $\left(R^{2}=.082\right)$. El Miedo Total supone el $\mathbf{2 8 . 6 \%}$ de esta varianza $(\beta=.286)$. Existe una relación inversamente proporcional entre el Miedo Total y las Habilidades Sociales.

Tabla 14 Regresión HHSS

$\begin{array}{ccccc}\text { Variables } & \text { B } & \text { Q } & \text { T } & \text { P } \\ \text { Constante General } & 99.802 & & 65.020 & .0000 \\ \text { Miedo Total } & -.097 & -.286 & -7.016 & .000\end{array}$

Habilidades Sociales= 99.802 - .097 (Miedo Total); R=.286; $R^{2}=.082 ; F=49.223$; $\mathrm{p}=.0000$; Fuente: elaboración propia

El principal predictor de la IE es la Edad, consecuentemente se obtuvieron mayores resultados en los grupos de mayor edad. Los mejores resultados (media: $\bar{X}=135.89$ ) los obtuvieron los del grado/ curso $2^{\circ}$ de Bachillerato, seguidos por $1^{\circ}$ de Bachillerato (131.93). Las diferencias son significativas para el Total $(p=.000)$, Autoconcepto $(p=.000)$, Empatía $(p=.000)$, Motivación $(p=.000)$, Conocimiento de sí mismos $(p=.000)$ y Autocontrol $(p=.000)$. Pero no resultaron significativas en el caso de Habilidades Sociales ( $p=.339)$. De acuerdo con lo anterior, a medida que se incrementa la Edad, también lo hace la IE.
Otro importante predictor de IE es el Estatus, en tanto se observó un incremento en las puntuaciones en los niveles más altos referentes al Estatus. El estatus alto ocupa la primera posición $(\bar{x}=127.77)$, seguido por el estatus medio (124.36), éste a su vez seguido por el estatus bajo (109.81), y por último el estatus medio-bajo (108.14). Las diferencias fueron estadísticamente significativas en IE Total $(p=.000)$, Autoconcepto $(p=.000)$, Empatía $(p=.006)$, Motivación $(p=.000)$, Conocimiento de sí mismos ( $p=.000)$ y Autocontrol $(p=.000)$, pero no lo fueron para las Habilidades Sociales $(p=.686)$. Se concluye que, a mayor estatus, mayores niveles de $\boldsymbol{I} \boldsymbol{E}$.

LaCultura actúa como predictor en la IE Totaly en algunosfactores. Los sujetos de Cultura/Religión cristiana presentaron niveles mayores en $\boldsymbol{I E}$, con puntuaciones más altas (123.06) que los musulmanes (114.55) en esta variable. En IE Total $(p=.000)$, Autoconcepto ( $p=.001)$, Empatía ( $p=.021$ ), Motivación ( $p=.004)$, Conocimiento de sí mismo $(p=.000)$ y Autocontrol $(p=.002)$ las diferencias fueron significativas, en tanto los cristianos obtuvieron mejores resultados en todos los casos. Sin embargo, este no fue el caso para las Habilidades Sociales $(p=0.999)$.

Las puntuaciones más altas las alcanzan los que reflejan bastante miedo ( $\bar{x}=118.96)$. Teniendo en cuenta la prueba ANOVA, se encontraron diferencias significativas en la IE Total $(p=.025)$, el Autoconcepto ( $p=.000)$, la Empatía $(p=.000)$, el Autocontrol $(p=.034)$, las Habilidades Sociales $(p=.000)$ y la Motivación $(p=.014)$. Por el contrario, las diferencias no son significativas para el Conocimiento de sí mismo ( $p=.282)$, factor en el que el Miedo no actúa como predictor.

\section{Discusión y conclusiones}

El mayor predictor de la variable Miedo es el género, siendo las mujeres (chicas) quienes presentaron niveles superiores. Lo anterior era de esperarse, teniendo en cuenta trabajos como el de Azpiazu, Esnaola y Sarasa (2015) y el de Gómez Ortiz, Casas y Ortega Ruiz (2016) especialmente este último que provee una posible explicación centrada en los patrones de educación diferencial que reciben niños y niñas, correspondiente a los diferentes estilos de crianza, partiendo o reforzando una concepción de las niñas como más emocionales que los varones. Esta realidad se aleja de las aproximaciones que destacan las diferencias físicas entre ambos géneros a la hora de explicar este y otros fenómenos (de Carvalho Pinto, Barros Dutra, Filgueiras, Pereira Juruena, \& Stingel, 2013).

La cultura actúa como predictor en un factor y al respecto fueron los musulmanes quienes presentaron niveles superiores para esta variable.

Como se comentó en el párrafo anterior, los estilos educativos de los padres y las madres guardan estrecha relación con el desarrollo de pautas de apego y, por ende, con el desarrollo de las habilidades emocionales. Así pues, estas diferencias en el estilo, propias de cada cultura, también se traducen en diferencias en los ajustes socioemocionales y la conducta de los sujetos en la etapa adolescente (Gómez Ortiz, Romera, \& Ortega Ruiz, 2015).

Los predictores para la IE son Edad, Estatus y Cultura. La Edad aparece en los totales y en los factores, incrementando su nivel con el Curso y actúa sólo sobre un factor, hecho que se evidencia en el trabajo de (Franco Nerin, Pérez Nieto, \& de Dios Pérez, 2014). Queda claro que el proceso de desarrollo emocional provoca importantes cambios en todas las capacidades que configuran la constelación de la Inteligencia Emocional, realidad ya descrita por varios autores (Cazalla Luna \& Molero, 2014).

Respecto al Estatus también existen diferencias evidentes, 


\section{inteligencia emocional adolescente}

Predicción a través del miedo

en tanto son superiores los resultados de los sujetos en niveles socioeconómicos superiores, conclusión que coincide con aquella expuesta en el trabajo de Gutiérrez y Expósito (2015).

En relación con este último predictor aparece la Cultura. Las personas pertenecientes a la Cultura/Religión cristiana-occidental fueron quienes presentaron niveles significativamente superiores en $\boldsymbol{I E}$, así como en las diferentes subcategorías (excepto $\boldsymbol{H H S S}$ ). De esta manera se corrobora el hecho que la cultura de origen influye en las relaciones afectivas y en la constitución de competencias emocionales (Gutiérrez \& Expósito, 2015), y para la población de caso en particular, el contexto autóctono fomenta un impulso para el desarrollo socioafectivo.

Finalmente, se reitera la existencia de una interacción entre el Miedo y la $\boldsymbol{I} \boldsymbol{E}$, y una relación directamente proporcional, en tanto incrementa la segunda a medida que incrementa el primero. Se concluye entonces que el Miedo funciona como predictor de la IE y de la mayoría de factores, situación que se presenta también en el caso de las HHSS, por lo tanto, el repertorio emocional de la persona influye claramente en sus habilidades socioemocionales (Ordóñez Camblor, y otros, 2014).

Por su parte, la percepción de Inteligencia Emocional demostró ser un buen predictor del adecuado funcionamiento personal, social y emocional del sujeto (Augusto Landa, López Zafra, \& Pulido Martos, 2011), así como uno de los principales predictores de la adaptación emocional ajustada y del bienestar personal (Limonero, Fernández Castro, Soler Oritja, \& Álvarez Moleiro, 2015), de modo que niveles altos en la percepción de IE son indicativos de un mejor ajuste psicológico, mayor salud mental, y mejor empleo de estrategias de afrontamiento más adaptativas (Cazalla Luna \& Molero, 2014).

Considerando todo lo anterior, este estudio resulta relevante y arroja luz sobre esta temática, con miras a que se desarrollen mejores entrenamientos de estas habilidades desde edades tempranas, los cuales puedan ser implementados en casa y, ante todo, ofrecidos en la escuela, procurando educar a los niños no sólo en capacidades intelectuales, sino también en las emocionales (Ferragut \& Fierro, 2012).

\section{Referencias}

Ascensio, M., Vila, M. G., Robles García, R., Páez, F., Fresán, A., \& Vázquez, L. (2012). Estudio de traducción, adaptación y evaluación psicométrica del Inventario de Miedos FSSC-II en una muestra de estudiantes de educación media superior. Salud Mental, 35, 195-203. Obtenido de http:// www.medigraphic.com/pdfs/salmen/sam-2012/sam123c.pdf

Augusto Landa, J. M., López Zafra, E., \& Pulido Martos, M. (2011). Inteligencia Emocional Percibida y estrategias de afrontamiento al estrés en profesores de enseñanza primaria: propuesta de un modelo explicativo con ecuaciones estructurales (SEM). Revista de Psicología Social, 226(3), 413-425. Obtenido de https://www.researchgate.net/ publication/233693489 Inteligencia Emocional...

Azpiazu, L., Esnaola, I., \& Sarasa, M. (2015). Predictive capacity of social support on emotional intelligence in adolescence. European Journal of Education and Psychology, 8(1), 23-29. DOI:10.1016/j.ejeps.2015.10.003

Balongo, E., \& Mérida, R. (2016). El clima de aula en los proyectos de trabajo. Perfiles Educativos, 38(152), 146-162. Obtenido de http://www.scielo.org. mx/pdf/peredu/v38n152/0185-2698-peredu-38-152-00146.pdf

Bazán López, J. L., Olórtegui Malaver, V. M., \& Huayanay Falconí, L. (2016). Prevalencia y factores asociados con la conducta suicida en adolescentes de Lima rural. Revista de Neuropsiquiatría, 79(1), 3-15. Obtenido de http://www.scielo.org.pe/scielo.php?pid=S0034$\underline{85972016000100002 \& \text { script }=\text { sci arttext }}$

Caballero Dominguez, C. C., Suarez Colorado, Y. P., \& Bruges Carbono, H. D. (2015). Characteristics of Emotional Intelligence in a Group of Higher Education Students with and without Suicidal Ideation. Revista CES Psicología, 8(2), 138-155. Obtenido de http://www.scielo.org.co/scielo.php?script=sci arttext\&pid $=\$ 2011-30802015000200008$
Cazalla Luna, N., \& Molero, D. (2014). Inteligencia emocional percibida, ansiedad y afectos en estudiantes universitarios. Revista Española de Orientación y Psicopedagogía, 25(3), 56-73. DOI:10.5944/reop.vol.25.num.3.2014.13858

Cruz, P. (2014). Creatividad e Inteligencia Emocional. Cómo desarrollar la competencia emocio ᄀnal, en Educación Infantil, a través de la expresión lingüística y corporal. Historia y Comunicación Social, 19, 107-118. Obtenido de https://revistas.ucm.es/HICS/article/viewFile/44944/42322

de Carvalho Pinto, B. M., Barros Dutra, N., Filgueiras, A., Pereira Juruena, M. F., \& Stingel, A. M. (2013). Diferenças de gênero entre universitários no reconhecimento de expressões faciais emocionais. Avances en Psicología Latinoamericana, 31(1), 200-222.

Obtenido de http://www.scielo.org.co/pdf/apl/v31n1/v31n1a17.pdf

Delgado, B., Inglés, C. J., \& García Fernández, J. M. (2013). Social anxiety and selfconcept in adolescence. Revista de Psicodidáctica, 18, 179-194. Obtenido de http://www.redalyc.org/html/175/17525667008/

Ferragut, M., \& Fierro, A. (2012). Inteligencia emocional, bienestar personal y rendimiento académico en preadolescentes. Revista Latinoamericana de Psicología, 44(3), 95-104.

Obtenido de http://www.redalyc.org/articulo.oa?id=80525022008

Ford, B., Mauss, I., Troy, A., Smolen, A., \& Hankin, B. (2014). Emotion Regulation Moderates the Risk Associated With the 5-HTT Gene and Stress in Children. Emotion, 14(5), 930 -939.

Obtenido de https://www.ncbi.nlm.nih.gov/pmc/articles/PMC4172506/

Franco Nerin, N., Pérez Nieto, M. Á., \& de Dios Pérez, M. J. (2014). Relación entre los estilos de crianza parental y el desarrollo de ansiedad y conductas disruptivas en niños de 3 a 6 años. Revista de Psicología Clínica con Niños y Adolescentes, 1(2), 149-156.

Obtenido de http://www.redalyc.org/articulo.oa?id=477147184006

Gómez Ortiz, O. D., Romera, M., \& Ortega Ruiz, R. (2015). Los estilos educativos paternos y maternos en la adolescencia y su relación con la resiliencia, el apego y la implicación en acoso escolar. Anales de psicología, 979-989. Obtenido de https://idus.us.es/xmlui/bitstream/handle/11441/48757

Gómez Ortiz, O., Casas, C., \& Ortega Ruiz, R. (2016). Ansiedad social en la adolescencia: factores psicoevolutivos y de contexto familiar. Behavioral Psychology/Psicología Conductual, 24(1), 29-49. Obtenido de https:// dialnet.unirioja.es/servlet/articulo?codigo $=5456785$

Gutiérrez, M., \& Expósito, J. (2015). Autoconcepto, dificultades interpersonales, habilidades sociales y conductas asertivas en adolescentes. vista Española de Orientación y Psicopedagogía, 26(2), 42-58. Obtenido de https://dialnet.unirioja.es/servlet/articulo?codigo=5224079

Knappe, S., Sasagawa, S., \& Creswell, C. (2015). Developmental epidemiology of social anxiety and social phobia in adolescents. En K. Ranta, A. La Greca, L. J. Garcia Lopez, \& M. Marttunen (Edits.), Social Anxiety and Phobia in Adolescents (págs. 39-70). Dresden [de]: Springer, Cham. DOI:10.1007/978-3-319-16703-9_3

Limonero, J. T., Fernández Castro, J., Soler Oritja, J., \& Álvarez Moleiro, M. (2015). Emotional intelligence and recovering from induced negative emotional state. Frontiers in Psychology, 6(816), 1-8. Obtenido de https://www.ncbi.n/m.nih.gov/pmc/articles/PMC4472988/

Ordóñez Camblor, N., Lemos Giráldez, S., Paino, M., Fonseca Pedrero, E., García Álvarez, L., \& Pizarro Ruiz, J. P. (2014). Relación entre psicosis y experiencias traumáticas tempranas. Anuario de Psicología, 44(3), 283294. Obtenido de http://www.redalyc.org/articulo.oa?id=97036176001

Pulido, F. (2016). El miedo como predictor del rendimiento académico: El contexto pluricultural de Ceuta. Revista Española de Orientación y Psicopedagogía, 27(2), 29-44.

Obtenido de http://revistas.uned.es/index.php/reop/article/view/17112

Pulido, F., \& Herrera, F. (2015). Miedo, Inteligencia Emocional y Rendimiento Académico en el contexto educativo pluricultural de Ceuta. Aprendiendo a Investigar: Aprendiendo a investigar, 1, 183-202.

Obtenido de https://dialnet.unirioja.es/servlet/tesis?codigo $=57730$

Ranøyen, I., Jozefiak, T., Wallander, J., Lydersen, S., \& Indredavik, M. S. (2014). Selfreported social anxiety symptoms and correlates in a clinical (CAP) and a community (Young-HUNT) adolescent sample. Social Psychiatry and Psychiatric Epidemiology, 49, 1937-1949.

Obtenido de https://www.ncbi.nlm.nih.gov/pubmed/24791658

Ruivo, J., \& Paes, S. (2015). Inteligencia emocional y liderazgo escolar. Aula, 21, 233244. Obtenido de http://ut.suagm.edu/sites/default/files/uploads/CentroEstudios-Doctorales/Tesis Doctorales/2014/BFlores.pdf

Sainz Gómez, M., Bermejo García, R., Ferrándiz García, C., Prieto Sánchez, M. D., \& Ruiz Melero, M. J. (2015). Cómo funcionan las competencias socioemocionales en los estudiantes de alta habilidad. Aula, 21, 33-47. Obtenido de http://revistas.usal.es/index.php/0214-3402/article/view/ aula2015213347

Sroufe, L. A. (2000). Desarrollo Emocional: La Organización de la Vida Emocional en los primeros años. (M. S. Donis Galindo, Trad.) Mexico DF [mx]: Oxford University Press. 